\title{
Time Localization of Transients with Wavelet Maxima Lines
}

\author{
Sylvain Meignen* \\ LMC-IMAG Laboratory, University of Grenoble, France \\ Tel:0033-4-76-51-43-95 \\ FAX:0033-4-76-63-12-63 \\ E-mail: sylvain.meignen@imag.fr \\ Sophie Achard \\ LMC-IMAG Laboratory, University of Grenoble, France \\ Tel:0033-4-76-63-58-29 \\ FAX:0033-4-76-63-12-63 \\ E-mail:sophie.achard@imag.fr \\ Pierre-Yves Guméry \\ TIMC-IMAG Laboratory, University of Grenoble, France \\ Tel:0033-4-76-63-74-55 \\ FAX:0033-4-76-63-71-86 \\ E-mail:gumery@imag.fr
}

\begin{abstract}
In this study, we present a new estimator of transients built from the extrema of a signal decomposition first introduced by Berkner. We study the performance of the new estimator of transients both for a variation of amplitude and a variation of frequency of the signal. We show that the time localization of transients is better estimated with the maxima lines made by the extrema in the time scale-space than with the wavelet coefficients computed with the CWT. We also verify that this improvement is not to the detriment of detection performance.
\end{abstract}

Keywords- estimation of transients, wavelet decomposition, maxima lines

EDICS Category:1-NPAR, 1-DETC 


\section{INTRODUCTION}

In this paper, we study the time localization of transients generated by either a variation of frequency or a variation of amplitude using wavelet extrema. We build a nonparametric estimator of transients which we compare to estimators that use wavelet coefficients. We do this both in terms of estimation and in terms of detection [9][6] on Gaussian signals, for the sake of simplicity.

The coefficients of wavelet transforms at different scales have been extensively used for change detection [9][6][1]. The robustness of the detector based on wavelets coefficients imposes to consider scales that correctly cover the frequency bandwidth of the signal [5]: the choice of pertinent scales is often a complicated issue. A second drawback of these methods is time delocalization of transients when the scale increases. The study of extrema of the signal decomposition over Gaussian or derivatives of Gaussian functions is a good mean to solve this problem. Indeed, extrema that arise from the convolution of the signal with Gaussian functions or derivatives of Gaussian functions are known to form connected curves in the time-scale space [12], called maxima lines, which link any singularity at a given scale to its origin at the finest scale. However, the practical construction of the maxima lines associated with these wavelet decompositions require ad-hoc procedures. We, therefore, use an approximation of the continuous wavelet transform [2] for which the construction of the maxima lines is mathematically defined. A second remaining problem is the choice of pertinent scales. After proving the completeness of the signal decomposition we use, we study the reconstruction of signals from extrema to derive automatically the pertinent scales to use. The construction of the estimator is based on the properties of maxima lines over the selected pertinent scales. Reference parameters are estimated over intervals without transients and then the quality of the estimator is tested through the computation of mean square errors and ROC curves.

The sketch of the article is as follows. We first recall convergence properties of discrete B-splines of which the signal decomposition we use is a particular case (section II). Section III is devoted to the completeness of the decomposition, while in section IV we define the maxima lines and the characteristic scale that arises from their signal reconstruction properties. Section $\mathrm{V}$ details the construction of the estimator of transients while a comparison with a wavelet coefficient based method concludes the paper.

\section{Convergence of Discrete B-Splines to GAUSSiAn FUnCtions}

In this section, we briefly recall the simple convergence properties of discrete B-splines to better understand the relation between the filters we will use and Gaussian functions. The continuous B-spline of order $n>0$ is:

$$
\beta^{n}(x)=\overbrace{\beta^{0} * \beta^{0} * \cdots * \beta^{0}}^{n+1}(x),
$$

where $\beta^{0}$ is the characteristic function of the interval $[0,1]$. The discrete $\mathrm{B}$-spline of order $n>0$, at scale $m$, is defined by [11]:

$$
b_{m}^{n}=\overbrace{b_{m}^{0} * b_{m}^{0} * \cdots * b_{m}^{0}}^{n+1},
$$


where $b_{m}^{0}=\frac{1}{m}\{1,1, \cdots, 1\}$ is the normalized sampled pulse of width $m \geq 2$. We also define $b_{m}^{-1}=\delta_{0}$ where $\left(\delta_{l}[p]\right)_{p \in \mathbb{Z}}$ is the sequence that equals 1 if $p=l$ and zero otherwise. The link between discrete and continuous B-splines is the dilation equation:

$$
\frac{1}{m} \beta^{n}\left(\frac{x}{m}\right)=b_{m}^{n} * \beta^{n}(x) .
$$

While B-splines at different scales $m$ involve a basis function with a regularity given by the order of the spline, the sequence of filters $b_{m}^{n}$ with fixed $m$ and large $n$ are approximations of a Gaussian function (i.e. $C^{\infty}$ ). Let $X$ denote a discrete random variable with uniform distribution over the set $\left\{-\frac{m-1}{2}, \cdots, \frac{m-1}{2}\right\}$ (resp. $\left\{-\frac{m}{2}+1, \cdots, \frac{m}{2}\right\}$ ) for $m$ odd (resp. even). Then $b_{m}^{n}[p]$ is the probability that the sum of $n+1$ independent identically distributed variables $X_{i}$ is equal to $p$ (since the law of a sum of independent identically distributed variables is obtained by convolution of their probability distributions). The mean of the variable $X_{i}$ is 0 (resp. $\frac{1}{2}$ ) if $m$ is odd (resp. even), while its standard deviation is $\sqrt{\frac{m^{2}-1}{12}}$. Applying the central limit theorem we get:

$$
\frac{\sum_{i=1}^{n+1} X_{i}-\epsilon \frac{n+1}{2}}{\sqrt{(n+1)\left(m^{2}-1\right) / 12}} \underset{n \rightarrow+\infty}{\rightarrow} N(0,1)
$$

in distribution, where $\epsilon=1$ if $m$ is even and 0 otherwise. We suppose that this is the case throughout the paper. This convergence property leads to the approximation for large $n$ :

$$
b_{m}^{n}[p] \approx \sqrt{\frac{6}{\pi(n+1)\left(m^{2}-1\right)}} \exp \left(\frac{6\left(p-\epsilon \frac{n+1}{2}\right)^{2}}{(n+1)\left(1-m^{2}\right)}\right) .
$$

To derive approximations of the derivatives of the Gaussian function, we consider $\rho=\{1,-1\}, \rho_{k}=\overbrace{\rho * \cdots * \rho}^{k}$, and $b_{m, k}^{n}=\rho_{k} * b_{m}^{n}$. With these notations, the following theorem holds:

Theorem 1: For large $n$ we have:

$$
b_{m, k}^{n}[p] \approx \sqrt{\frac{6}{\pi(n+1)\left(m^{2}-1\right)}}\left[\exp \left(\frac{6 x^{2}}{(n+1)\left(1-m^{2}\right)}\right)\right]^{(k)}\left(p-\frac{\epsilon(n+1)+k}{2}\right)
$$

This result is proved in Appendix A. We recall that $n$ is the order of the B-spline, $\frac{1}{m}$ is the sampling rate of the interval $\left[-\frac{1}{2}, \frac{1}{2}\right]$ and $k$ is the order of the derivative. To get an approximation of the $k$ th derivative of the Gaussian one must shift the filter $b_{m, k}^{n}$ properly ; we define:

$$
\alpha_{m, k}^{n}[p]=b_{m, k}^{n}\left[p+\left\lfloor\frac{\epsilon(n+1)+k}{2}\right\rfloor\right]
$$

where $\lfloor$.$\rfloor denotes the integer part, to obtain unshifted approximations of the derivatives of Gaussian functions.$

\section{ReCONSTRUCtion PROPERTy OF THE FILTERS $\alpha_{m, k}^{n}$}

By analogy with the wavelet decomposition which uses reverse time version of the wavelets, we consider the correlation of the sequence $f[j]$ with $\alpha_{m, k}^{n}[j]$ defined in (4):

$$
\begin{aligned}
\forall p \in \mathbb{Z} \quad c_{m, k}^{n}[p] & =\sum_{j \in \mathbb{Z}} \alpha_{m, k}^{n}[j] f[j+p] \\
& =\sum_{j \in \mathbb{Z}} \alpha_{m, k}^{n}[j-p] f[j] \text { for } n \geq-1
\end{aligned}
$$


The family $G=\left\{\alpha_{m, k}^{n, p}[j]=\alpha_{m, k}^{n}[j-p], p \in \mathbb{Z}, N>n \geq-1\right\}$ is a frame of $l_{2}(\mathbb{Z})$ if and only if the Fourier series of $\alpha_{m, k}^{n}$ for $N>n \geq-1$ do not have a common zero in ] $\left.-\frac{1}{2}, \frac{1}{2}\right]$ ([10],Corollary 3). As $\alpha_{m, k}^{n}[p]$ is a shifted version of $b_{m, k}^{n}[p]$ (see (4)) both Fourier series have the same modulus which is given by:

$$
\left|\hat{\alpha}_{m, k}^{n}(\nu)\right|=2^{k}|\sin (\pi \nu)|^{k}\left|\frac{\sin _{c}(\pi m \nu)}{\sin _{c}(\pi \nu)}\right|^{n+1}
$$

If $k>0$ (the case $k=0$ is uninteresting) and for $N \geq n \geq 0$ the Fourier series is null at $\nu \in\left\{\left|\frac{l}{m}\right| \leq \frac{1}{2}, l \in \mathbb{Z}\right\}$ and if $n=-1$ the Fourier series is null at $\nu=0$. The family $G$ therefore does not satisfy the frame condition if $k>0$. However, if we add to $G$ the complementary sequence $\alpha_{m, k^{\prime}}^{N, p}, 0 \leq k^{\prime}<k$ we have a frame since the Fourier series of $\alpha_{m, 0}^{N, p}$ is not null for $\nu=0$. Although this construction is not optimal in terms of number of basis filters, it will prove to be relevant for our purpose. From the frame theory, the signal $f$ can then be expanded as [4]:

$$
f[l]=\sum_{0 \leq k^{\prime}<k} \sum_{p \in \mathbb{Z}} c_{m, k^{\prime}}^{N}[p] \tilde{\alpha}_{m, k^{\prime}}^{N, p}[l]+\sum_{n=-1}^{N-1} \sum_{p \in \mathbb{Z}} c_{m, k}^{n}[p] \tilde{\alpha}_{m, k}^{n, p}[l]
$$

where . denotes the frame dual to

$$
F_{m, k}^{N}=\left\{\alpha_{m, k^{\prime}}^{N, p}, 0 \leq k^{\prime}<k, \alpha_{m, k}^{n, p},-1 \leq n<N, p \in \mathbb{Z}\right\} .
$$

The formula (5) ensures that the reconstruction of $f$ with the coefficients of the decomposition is always possible but may be complicated. However for $m=2$, we show that we have a simple and explicit formula. Indeed, recall that the sequence $c_{2, k}^{n}$ satisfies:

$$
\text { if } \begin{aligned}
n+k \text { is odd, } c_{2, k}^{n}[p] & =\frac{1}{2}\left(c_{2, k}^{n-1}[p-1]+c_{2, k}^{n-1}[p]\right) \\
c_{2, k}^{n}[p] & =c_{2, k-1}^{n}[p-1]-c_{2, k-1}^{n}[p] \\
\text { else, } c_{2, k}^{n}[p] & =\frac{1}{2}\left(c_{2, k}^{n-1}[p]+c_{2, k}^{n-1}[p+1]\right) \\
c_{2, k}^{n}[p] & =c_{2, k-1}^{n}[p]-c_{2, k-1}^{n}[p+1]
\end{aligned}
$$

which leads to:

$$
\begin{gathered}
\frac{1}{2} c_{2, k}^{n-1}[p]=c_{2, k-1}^{n-1}[p]-c_{2, k-1}^{n}[p] \quad n+k \text { odd } \\
\frac{1}{2} c_{2, k}^{n-1}[p]=c_{2, k-1}^{n-1}[p-1]-c_{2, k-1}^{n}[p] \text { otherwise }
\end{gathered}
$$

and finally, for $-1 \leq l \leq N-1$ :

$$
\begin{aligned}
c_{2, k-1}^{l}[p]= & c_{2, k-1}^{N}\left[p+\left\lfloor\frac{N-l+1}{2}\right\rfloor\right] \\
& +\frac{1}{2} \sum_{n=l}^{N-1} c_{2, k}^{n}\left[p+\left\lfloor\frac{n-l+1}{2}\right\rfloor\right] \quad k \text { odd } \\
c_{2, k-1}^{l}[p]= & c_{2, k-1}^{N}\left[p+\left\lfloor\frac{N-l+1}{2}\right\rfloor\right] \\
& +\frac{1}{2} \sum_{n=l}^{N-1} c_{2, k}^{n}\left[p+\left\lfloor\frac{n-l+2}{2}\right\rfloor\right\rfloor \text { otherwise. }
\end{aligned}
$$


(6) is proved in Appendix B. Property (7) is a direct consequence of (6) and leads to (8). Property (8) gives a simple procedure for reconstructing the signal from the decomposition over $F_{2, k}^{N}$. Indeed, if we assume the decomposition over $F_{2, k}^{N}$ to be known, we get the coefficients $c_{2, k-1}^{l}[p]$ for $-1 \leq l \leq N-1$, the coefficients $c_{2, k-1}^{N}[p]$ being already known because $\alpha_{2, k-1}^{N, p}$ belongs to $F_{2, k}^{N}$. If we apply the procedure $k$ times, we get the coefficients $c_{2,0}^{l}[p]$, for $-1 \leq l \leq N-1$, in particular for $l=-1$ we get the signal $f[p]$.

\section{Definition of Maxima Lines, Signal Reconstruction From Extrema}

\section{A. Definition of Maxima Lines}

We define the maxima of the sequence $c_{m, k}^{n}$ (respectively minima) as the strictly positive (respectively negative) coefficients $c_{m, k}^{n}[p]$ such that $c_{m, k}^{n}[p-1]<c_{m, k}^{n}[p]>c_{m, k}^{n}[p+1]\left(\operatorname{resp} . c_{m, k}^{n}[p-1]>c_{m, k}^{n}[p]<c_{m, k}^{n}[p+1]\right)$. The specificity of the case $m=2$ is that an extremum at rank $n$ arises from a unique extremum of the same nature at rank $n-1$. These extrema define curves in the time-scale space which are called maxima lines. The practical construction of maxima lines in this case is explained in [2] [3] [8] and works for any signal. On the contrary, for $m>2$, recalling that the sequence $c_{m, k}^{n}$ is obtained by correlation of $c_{m, k}^{n-1}$ with a shifted version of $b_{m}^{0}$ (see (5)), if $c_{m, k}^{n-1}$ contains a sequence of the kind $0 \cdots 01 \overbrace{0 \cdots 0}^{m-2} 10 \cdots 0$ with two maxima, the correlation with $b_{m}^{0}$ will lead to a unique maximum for $c_{m, k}^{n}$ which arises from the two extrema at rank $n-1$. Consequently, the maximum at rank $n$ is associated with two maxima at rank $n-1$ which entails different possible constructions for the maxima lines. For that reason, we will take $m=2$ for the construction of maxima lines.

\section{B. Signal Reconstruction from Extrema when $k=1$}

We study here the reconstruction of the signal $f$ from the extrema of the sequence $c_{2,1}^{n}$, when $f$ has zero mean without loss of generality. In such a case, the origin of the maxima lines (i.e. for $n=-1$ ) corresponds to a maximum of the derivative of the signal (i.e. a change of curvature). The sequence $c_{2,0}^{N}[p]$ tends to zero when $N$ tends to infinity (we do not prove it here due to the lack of space; see [8] for details). Since $c_{2,0}^{-1}[p]=f[p]$, we derive using (8):

$$
f[p] \approx \frac{1}{2} \sum_{n=-1}^{N-1} c_{2,1}^{n}\left[p+\left\lfloor\frac{n+2}{2}\right\rfloor\right]
$$

We define $\bar{c}_{2,1}^{n}[p]$ by $c_{2,1}^{n}[p]$ if there is an extremum in $p$ at rank $n$ and zero otherwise. Let us denote $g_{N}$ by

$$
g_{N}[p]=\frac{1}{2} \sum_{n=-1}^{N} \bar{c}_{2,1}^{n}\left[p+\left\lfloor\frac{n+2}{2}\right\rfloor\right]
$$

and also $E_{N}$, the reconstruction error from extrema, by

$$
E_{N}=\sum_{p \in Z}\left(f[p]-g_{N}[p]\right)^{2}
$$

We have carried out simulations to estimate the evolution of $E_{N}$ for signals with different frequency bandwidths. The signals we used in simulations were generated through the filtering of uniform noise by Butterworth filters with various frequency bandwidths. We notice on the simulated signals that the reconstruction error $E_{N}$ decreases with 
$N$ for small $N$, then reaches a minimum ( the corresponding $N$ is called $N_{0}$ ) and finally stabilizes. The value $N_{0}$ associated with the minimal reconstruction error is larger when the frequency bandwidth is low, since in that case neighboring extrema are, on average, further apart. In Figure 1, we display the typical evolution of the square root of $E_{N}$ normalized by the variance of $f$ (i.e. $\sqrt{\sum_{p \in \mathbb{Z}}(f[p])^{2}}$ ) for three signals with different frequency bandwidths.

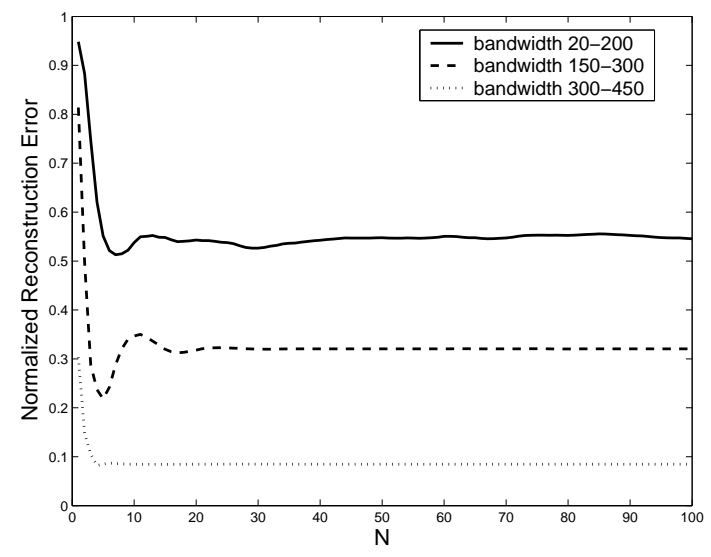

Fig. 1. Computation of the normalized reconstruction errors for three signals with bandwidths $20-200 \mathrm{~Hz}, 100-350 \mathrm{~Hz}, 300-450 \mathrm{~Hz}$, the sampling frequency being $1 \mathrm{kHz}$

The theoretical demonstration of the behavior of $E_{N}$ is not simple; we only give a numerical insight of what is happening. The error satisfies the induction property:

$$
\begin{array}{r}
E_{N}=E_{N-1} \\
-\frac{1}{2} \sum_{p \in \mathbb{Z}}\left(f[p]-\frac{1}{2} \sum_{n=-1}^{N-2} \bar{c}_{2,1}^{n}\left[p+\left\lfloor\frac{n+2}{2}\right\rfloor\right]\right) \bar{c}_{2,1}^{N-1}\left[p+\left\lfloor\frac{N+1}{2}\right\rfloor\right] \\
+\frac{1}{4} \sum_{p \in \mathbb{Z}} \bar{c}_{2,1}^{N-1}\left[p+\left\lfloor\frac{N+1}{2}\right\rfloor\right]^{2}
\end{array}
$$

The decay of the error is linked to the second term on the right side of the equality. For a small $N$, when $f[p]-\frac{1}{2} \sum_{n=-1}^{N-2} \bar{c}_{2,1}^{n}\left[p+\left\lfloor\frac{n+2}{2}\right\rfloor\right]$ and $\bar{c}_{2,1}^{N-1}\left[p+\left\lfloor\frac{N+1}{2}\right\rfloor\right]$ are not null, for most of the time they have the same sign. This is due to the fact that the first term has the sign of $f$ at $p$ and that a maximum for $c_{2,1}^{N-1}$ is more likely to occur when $f$ is positive (the opposite is true for a minimum). When $N$ increases the shift $\left\lfloor\frac{N+1}{2}\right\rfloor$ makes $c_{2,1}^{N-1}\left[p+\left\lfloor\frac{N+1}{2}\right\rfloor\right]$ correspond to a minimum when it initially corresponded to a maximum and thus it changes signs, while $f[p]-\frac{1}{2} \sum_{n=-1}^{N-2} \bar{c}_{2,1}^{n}\left[p+\left\lfloor\frac{n+2}{2}\right\rfloor\right]$ keeps the same sign (the reasoning is also true in the reverse case). The effect of this change of sign is an increase of the mean square error for a value $N_{0}$ of $N$.

\section{Construction of the Estimator of Transients}

In this section, we first build a new nonparametric estimator of transients using the maxima lines of the decomposition (with $k=1$ and $m=2$ ). The ranks $n$ we consider, to build the estimator, are lower than $N_{0}$, 
determined for each signal independently using the reconstruction procedure detailed above. We first explain how we characterize a signal with its maxima lines, then how to compute the reference parameters on an interval supposed to be free of transients. In a second time, we introduce the test statistics on the maxima to detect transients and estimate their localization. Finally, we recall the principle of transient estimation with wavelet coefficients under the Gaussian hypothesis.

\section{A. Signal Characterization}

As each extremum of the sequence $c_{2,1}^{n}$ belongs to a single maxima line (see definition in section IV.A) [2] [3] [8], we can associate with each maxima line $\mathcal{L}$ in the time-scale space (where the time is indexed by $p$ and the scale by $n$ ) the variable $D_{\mathcal{L}, q}$, with $|\mathcal{L}| \geq q+2$ (where $|\mathcal{L}|$ is the length of $\mathcal{L}$ ) defined by:

$$
D_{\mathcal{L}, q}=\sum_{(p, n) \in \mathcal{L}, n \leq q} \frac{\left(c_{2,1}^{n}[p]\right)^{2}}{\left\|\alpha_{2,1}^{n}\right\|_{2}^{2}}
$$

and $\left\|\alpha_{2,1}^{n}\right\|_{2}=\sqrt{\sum_{p \in \mathbb{Z}}\left(\alpha_{2,1}^{n}[p]\right)^{2}}$. The role of the normalization is to give the same relative importance to each coefficient $\left(c_{2,1}^{n}\right)^{2}$ in the sum (the $\alpha_{2,1}^{n}$ are then normalized to 1 in the $l_{2}$ sense).

The variable $D_{\mathcal{L}, q}$ is not sufficient to properly characterize frequencies of the signal. We therefore use another variable:

$$
F_{\mathcal{L}, q}=O\left(\mathcal{L}_{+}(q)\right)-O(\mathcal{L})
$$

where $O(\mathcal{L})$ is the origin of the maxima line $\mathcal{L}$ and $\mathcal{L}_{+}(q)$ is the maxima line that follows $\mathcal{L}$ at rank $q$.

\section{B. Principle of the Estimation-Detection}

We compute the variable $D_{\mathcal{L}, q}$ when $q \leq N_{0}$ (see section IV. B for the definition of $N_{0}$ ) for a reference part of $f$ assumed to be free of transients. For any probability $\operatorname{Pr}$, the empirical distribution of $D_{\mathcal{L}, q}$ provides thresholds $a_{q}$ and $b_{q}$ such that $P\left(a_{q}<D_{\mathcal{L}, q}<b_{q}\right)=\operatorname{Pr}$. For each maxima line $\mathcal{L}$ such that $|\mathcal{L}| \geq q+2$ we have the standard choice between:

$$
\begin{gathered}
H_{0}(q): D_{\mathcal{L}, q} \text { is in }\left[a_{q}, b_{q}\right] \\
H_{1}(q): D_{\mathcal{L}, q} \text { is out of }\left[a_{q}, b_{q}\right]
\end{gathered}
$$

The variable $F_{\mathcal{L}, q}$ takes integer values and we compute its distribution for each $q \leq N_{0}$. Any probability $\operatorname{Pr}$ defines a subset $A(q)$ of $\mathbb{N}$ such that $A(q)=\left\{x, P\left(F_{\mathcal{L}, q}=x\right)>1-\operatorname{Pr}\right\}$. For each maxima line, we again have the choice between:

$$
\begin{gathered}
H_{0}^{\prime}(q): F_{\mathcal{L}, q} \text { is in } A(q) \\
H_{1}^{\prime}(q): F_{\mathcal{L}, q} \text { is not in } A(q)
\end{gathered}
$$

To summarize, once we are given a probability $\operatorname{Pr}$, we can compute $a_{q}, b_{q}$ and $A(q),-1 \leq q \leq N_{0}$ which we use in the estimation. Note that from a detection point of view $1-P r$ is the probability of false alarm.

We now assume that for any probability $\operatorname{Pr}$ reference features $A(q), a_{q}$ and $b_{q},-1 \leq q \leq N_{0}$ have been computed on $[0, T-d]$ considered as the reference part of the signal where $T$ is the transition time and $d$ defines the maximum distance from the true transition $T$. 
We build a nonparametric test to estimate the transition time using the maxima lines. We consider the maxima lines $\mathcal{L}$ such that $O(\mathcal{L})$ is inside $[T-d ; T+d]$. If $|\mathcal{L}| \geq q+2$, four cases may occur:
i) $\mathcal{L}$ satisfies $H_{0}(q) \cup H_{0}^{\prime}(q)$
ii) $\mathcal{L}$ satisfies $H_{1}(q) \cup H_{0}^{\prime}(q)$
iii) $\mathcal{L}$ satisfies $H_{0}(q) \cup H_{1}^{\prime}(q)$
iv) $\mathcal{L}$ satisfies $H_{1}(q) \cup H_{1}^{\prime}(q)$

We then scan the interval $[T-d, T+d]$. The first line $\mathcal{L}$ that satisfies hypothesis ii) or iv) at rank $q$ corresponds to a transient $T_{1}(q)=O(\mathcal{L})$, while the first line $\mathcal{L}$ that satisfies iii) or iv) corresponds to a transient $T_{2}(q)=O(\mathcal{L})$. We thus have two vectors $T_{1}$ and $T_{2}$ for which the best ranks $q$ are those that maximize the probability of transition, i.e. the probability that the line $\mathcal{L}$ does not correspond to the known part of the signal defined for $t \leq T-d$. If we denote $q_{1}$ (resp. $q_{2}$ ) the rank associated with $T_{1}$ (resp. $T_{2}$ ), we choose between $T_{1}\left(q_{1}\right)$ and $T_{2}\left(q_{2}\right)$ taking the one with the highest probability of transition. If we denote $P(\tilde{T})$ the probability of transition of $\tilde{T}$, the estimated transition $\hat{T}$ is:

$$
\begin{aligned}
& \hat{T}=\operatorname{argmax}\left\{P(\tilde{T}), \tilde{T} \in\left\{\quad T_{1}\left(\underset{q \leq N_{0}}{\operatorname{argmax}} P\left(T_{1}(q)\right)\right),\right.\right. \\
& \left.\left.\left.T_{2} \underset{q \leq N_{0}}{\operatorname{argmax}} P\left(T_{2}(q)\right)\right)\right\}\right\}
\end{aligned}
$$

As the estimated transition time is associated with a maxima line generated by an extremum of the derivative of the signal, the detected transition always correspond to a variation of curvature of the signal.

\section{Continuous Wavelet Decomposition and Estimation of Transients in Gaussian Signals [6]}

In order to make a comparison, we recall the principle of transient estimation with wavelet coefficients. Suppose that a signal $f$ is decomposed onto a family of wavelets of the kind:

$$
\left\{\Psi_{s, b}(t)=\frac{1}{s} \Psi\left(\frac{t-b}{s}\right), 1 \leq s \leq S\right\}
$$

If $f$ is Gaussian, $Y[b]=\left(Y_{s}[b]=\left(f(t), \frac{1}{s} \Psi\left(\frac{t-b}{s}\right)\right)\right)_{s \leq S}$ is a Gaussian vector with zero mean components characterized by its covariance matrix $\sum$. In such a case, $Z[b]=Y[b]\left(\sum\right)^{-1} Y[b]$ is $\chi_{2}$ distributed with $S$ degrees of freedom [6]. As previously, we seek, for different values of $S$, the first time in $[T-d, T+d]$ when $Z$ does not satisfy the $\chi_{2}$ hypothesis for any probability $\operatorname{Pr}$. Consequently, the comparison of our estimator of transients to that based on wavelet coefficients is easily carried out on Gaussian signals.

\section{Results}

The signals we consider are Gaussian white noises filtered by Butterworth filters which are Gaussian signals to enable simple comparison between the different methods. We study two kinds of transitions: first, a variation of amplitude of the signal and, second, a variation of frequency. Each signal has a sample rate of $1 \mathrm{kHz}$. The true transition is in all cases $T=800 \mathrm{~ms}$; we fix $d=10 \mathrm{~ms}$. We consider $T-d$ very large to ensure that the reference 
parameters are correctly estimated. The choice for the value of $d$ is justified by EMG practical applications: we are to use the estimator to measure EMG (electromyographic) latency responses of muscles under stimulation whose average value is known. Then, the interval where the transients should be detected needs not be too large. Furthermore, this practical assumption will improve the estimator performance because choosing a larger interval may add spurious detections that would alter the measurement of the estimator performance. For the method based on wavelet coefficients, we use the derivative of the normalized Gaussian $\Psi(x)=-\frac{x}{\sqrt{2 \pi}} \exp \left(-\frac{x^{2}}{2}\right)$ to be coherent with the choice $k=1$ of our decomposition. For this method, scale selection is often made considering that the family of wavelets ensures a good coverage of the frequency bandwidth of the signal [5]. We will see that this method leads to a worse estimation of transients than that which we propose.

\section{A. Variation of Amplitude}

The variation of amplitude is generated through a multiplication of the signal by $\beta$ at time $T$. We consider signals with frequency bandwidths $20-400 \mathrm{~Hz}$ to nearly cover the whole frequency spectrum (the sampling rate is $1 \mathrm{kHz}$ ). For these signals, we build ROC curves (detection versus false alarm) for $\beta=3$ and $\beta=1.5$, for our model (ML) and for the wavelet method for $S=2, S=4$ and $S=10$ and the corresponding mean square errors (the curves are build from 500 Monte Carlo runs). The results are depicted in Figure 2 in which we notice that the transient estimation (second row of the Figure) is approximately invariant with respect to the probability of false alarm. In fact, with the ML method, when a transient is detected at rank $n$ we can come back to its initial location (i.e rank $n=-1$ ) following the corresponding maxima line. On the contrary, with the wavelet methods, the wavelet coefficients at scale $s$ and time $t$ mix the information around $t$ introducing a bias in the estimation which is more important when $S$ is large. We also note that in the studied cases, the improvement of the estimation is not made to the detriment of the detection since the ROC curves for the ML method and for the wavelet method are very similar.

\section{B. Variation of Frequency}

We investigate the estimator of transients when the latter arises from a variation of frequency. At time $T$, we change the frequency bandwidth of the Butterworth filter. We study two cases: the first one is when the bandwidth varies from $20-200 \mathrm{~Hz}$ to $200-400 \mathrm{~Hz}$, while the second is the opposite. We build ROC curves of the detector associated with the $M L$ method and the wavelet method (for $S=2, S=4, S=10$ ) and the mean square errors to evaluate the performance of the estimators (the curves are derived from 500 Monte Carlo runs). The results are very similar to that obtained for a variation of frequency, i.e. while the estimator computed from wavelet coefficients are very sensitive to the probability of false alarm, the performance of the estimator based on maxima lines is invariant with respect to this parameter (see Figure 3). For the transition from $200-400 \mathrm{~Hz}$ to $20-200 \mathrm{~Hz}$, we notice that the ML method is slightly less performant in terms of detection than those based on wavelet coefficients, the reason is that $d$ is small compared to the lowest frequency $(20 \mathrm{~Hz})$ and therefore few maxima lines have their origin in the interval $[T-d, T+d]$. 


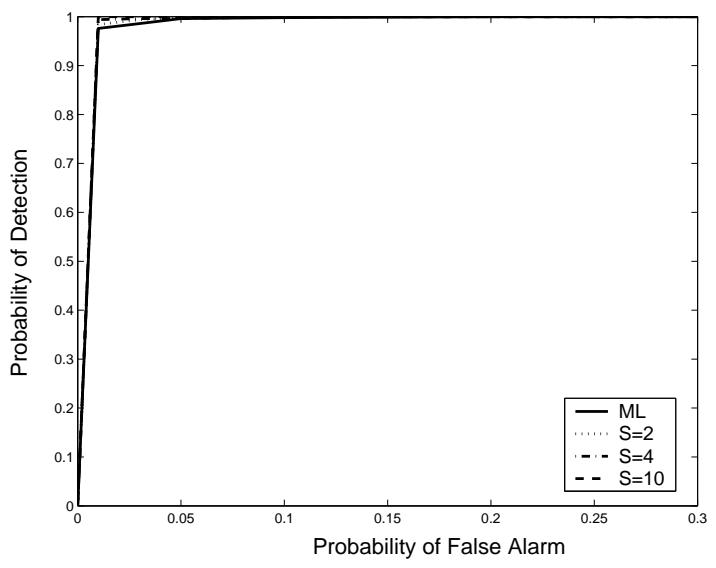

(A)

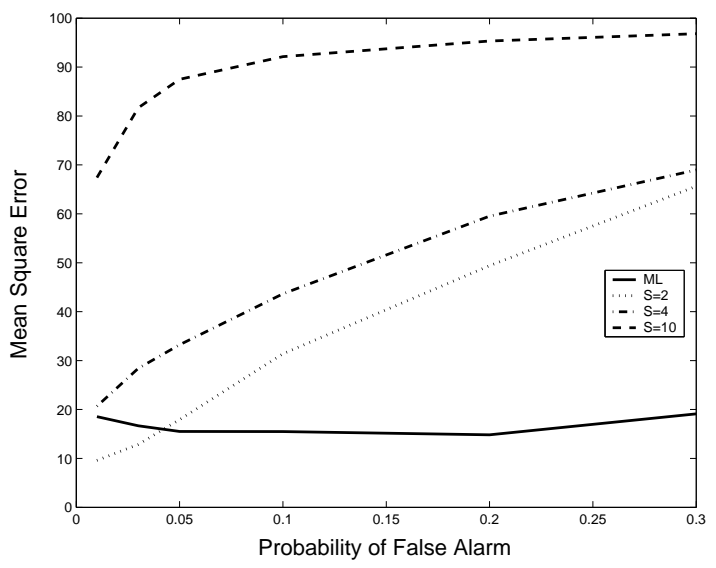

(C)

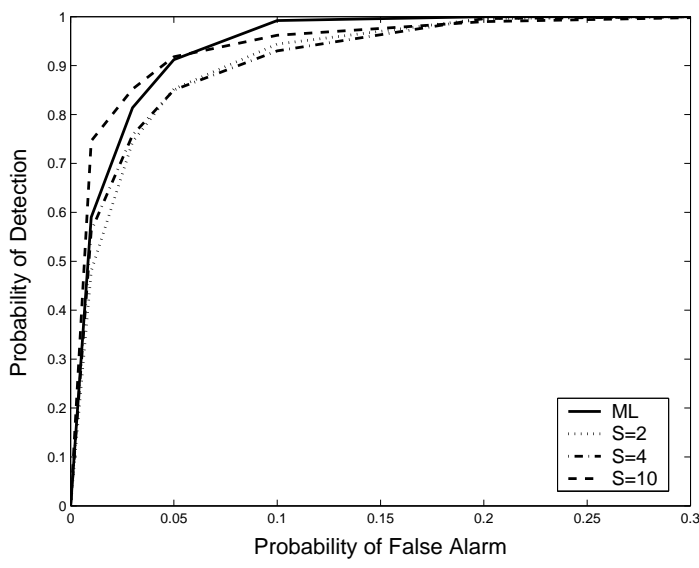

(B)

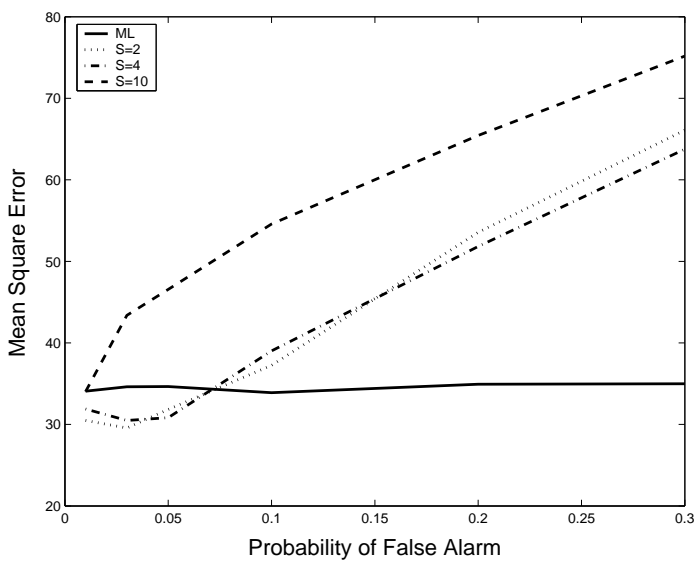

(D)

Fig. 2. (A):ROC curves for $\beta=3$ and a bandwidth $20-400 \mathrm{~Hz}$ for ML method and wavelet methods for $S=2, S=4$ and $S=10$. (B): idem but for $\beta=1.5$. (C) Mean square error for $\beta=3$ and a bandwidth $20-400 \mathrm{~Hz}$ for ML method and wavelet methods for $S=2, S=4$ and $S=10$. (D):idem but for $\beta=1.5$

\section{CONCLUSION}

The aim of this study was to introduce a new estimator of transients. The study of the maxima lines associated with the decomposition introduced by Berkner [2] on Gaussian signals suggested specific scales which are useful for the construction of a new estimator of transients. The latter is not parametric and mixes two characteristics to take both amplitude and frequency variations into account. It is a better estimator than the estimator based on wavelet coefficients computed using the CWT without any loss of detection performance. Another advantage of the method is that the pertinent scale selection is automatic which is not the case for the method that uses wavelet coefficients. Future work should consist in applying the proposed estimator of transients to the nongaussian and nonstationary cases. We also have to see the influence of maxima lines of a higher degree (i.e. $k>1$ ) on the estimation performance. Another direction for future work would also be to investigate the impact of noise on the 


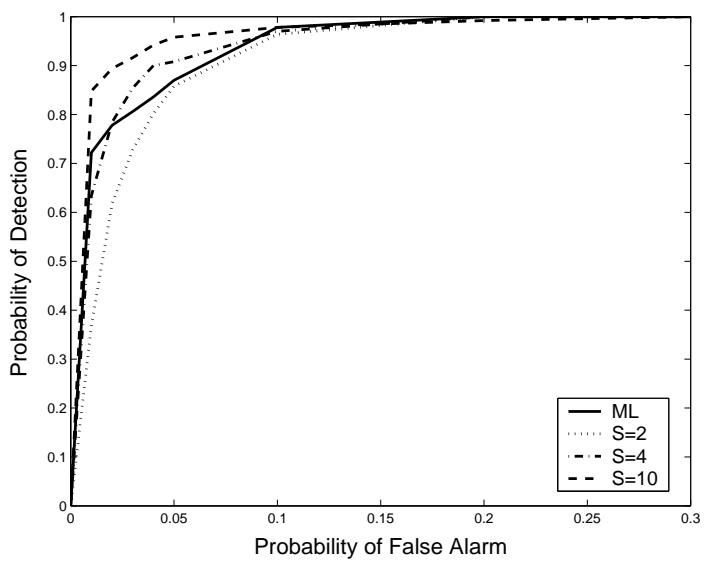

(A)

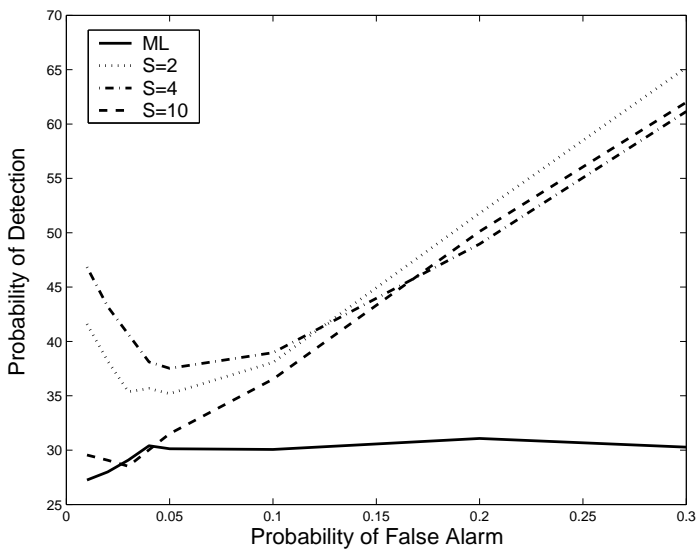

(C)

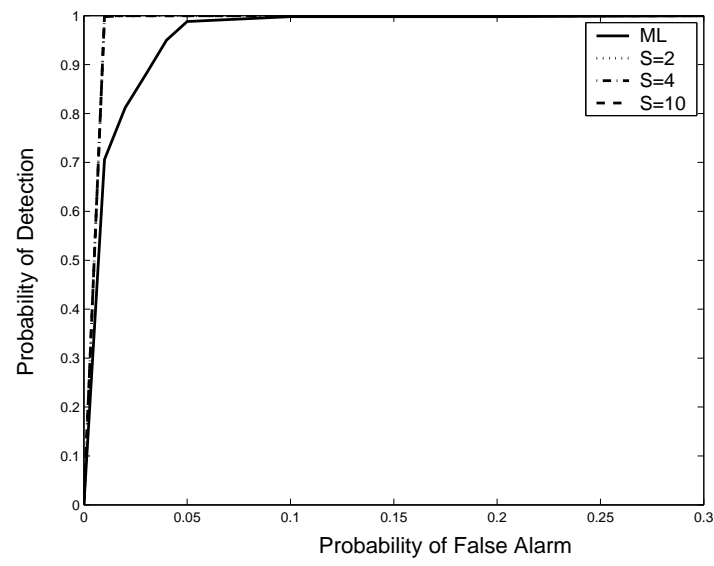

(B)

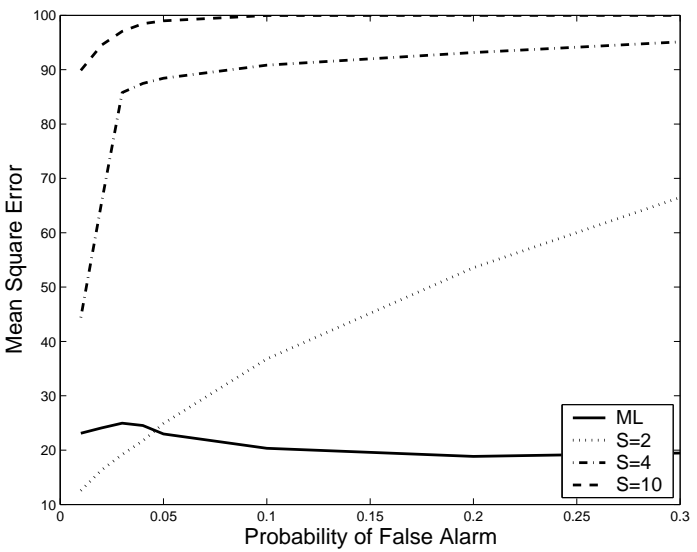

(D)

Fig. 3. (A): ROC curves for ML method and wavelet methods $(S=2, S=4, S=10)$ for a variation of frequency bandwidth from $20-200 H z$ to $200-400 \mathrm{~Hz}$, (B): idem but for a variation of frequency from $200-400 \mathrm{~Hz}$ to $20-200 \mathrm{~Hz}$, (C): Mean square error for the estimation of transition for a variation from $20-200 \mathrm{~Hz}$ to $200-400 \mathrm{~Hz}$, (D): idem for a variation from $200-400 \mathrm{~Hz}$ to $20-200 \mathrm{~Hz}$

estimator performance.

\section{APPENDIX A}

Proof OF THEOREM 1. it is made by induction on $\mathrm{k}$. We distinguish the cases $m$ even and $m$ odd. If $m$ is odd we can write:

$$
\begin{array}{r}
b_{m, 1}^{n}[p]=b_{m}^{n}[p]-b_{m}^{n}[p-1] \approx \sqrt{\frac{6}{\pi(n+1)\left(m^{2}-1\right)}} \times \\
\left(\exp \left(\frac{6 p^{2}}{(n+1)\left(1-m^{2}\right)}\right)-\exp \left(\frac{6(p-1)^{2}}{(n+1)\left(1-m^{2}\right)}\right)\right) \\
=\sqrt{\frac{6}{\pi(n+1)\left(m^{2}-1\right)}} \exp \left(\frac{6\left(p-\frac{1}{2}\right)^{2}}{(n+1)\left(1-m^{2}\right)}\right) \times
\end{array}
$$




$$
\begin{aligned}
& \left(\exp \left(\frac{6\left(p-\frac{1}{4}\right)}{(n+1)\left(1-m^{2}\right)}-\exp \left(\frac{-6\left(p-\frac{3}{4}\right)}{(n+1)\left(1-m^{2}\right)}\right)\right)\right. \\
& \approx \sqrt{\frac{6}{\pi(n+1)\left(m^{2}-1\right)}} \frac{12 p-6}{(n+1)\left(1-m^{2}\right)} \exp \left(\frac{6\left(p-\frac{1}{2}\right)^{2}}{(n+1)\left(1-m^{2}\right)}\right),
\end{aligned}
$$

using a first order approximation of the exponential in 0 ,

$$
=\sqrt{\frac{6}{\pi(n+1)\left(m^{2}-1\right)}}\left[\exp \left(\frac{6 x^{2}}{(n+1)\left(1-m^{2}\right)}\right)\right]^{\prime}\left(p-\epsilon \frac{n+1}{2}-\frac{1}{2}\right) .
$$

When $m$ is even, we have:

$$
\begin{array}{r}
b_{m, 1}^{n}[p]=b_{m}^{n}[p]-b_{m}^{n}[p-1] \approx \sqrt{\frac{6}{\pi(n+1)\left(m^{2}-1\right)}} \times \\
\left(\exp \left(\frac{6\left(p-\frac{(n+1)}{2}\right)^{2}}{(n+1)\left(1-m^{2}\right)}\right)-\exp \left(\frac{6\left(p-1-\frac{(n+1)}{2}\right)^{2}}{(n+1)\left(1-m^{2}\right)}\right)\right) \\
=\sqrt{\frac{6}{\pi(n+1)\left(m^{2}-1\right)}} \frac{12 \alpha}{\pi(n+1)\left(1-m^{2}\right)} \exp \left(\frac{6 \alpha^{2}}{(n+1)\left(1-m^{2}\right)}\right), \\
\approx \sqrt{\frac{6}{\pi(n+1)\left(m^{2}-1\right)}}\left[\exp \left(\frac{6 x^{2}}{(n+1)\left(1-m^{2}\right)}\right)\right]^{\prime}\left(p-\frac{n+1}{2}-\frac{1}{2}\right) .
\end{array}
$$

We now assume that at rank $k$ :

$$
\begin{array}{r}
b_{m, k}^{n}[p] \approx \sqrt{\frac{6}{\pi(n+1)\left(m^{2}-1\right)}} \times \\
{\left[\exp \left(\frac{6 x^{2}}{(n+1)\left(1-m^{2}\right)}\right)\right]^{(k)}\left(p-\frac{\epsilon(n+1)+k}{2}\right),}
\end{array}
$$

with $\epsilon=1$ if $m$ is odd and 0 otherwise. We first consider the case $m$ odd. Note that the derivative at rank $k$ of $\exp \left(\frac{6 x^{2}}{(n+1)\left(1-m^{2}\right)}\right)$ can be written as $P\left(\frac{x}{(n+1)\left(1-m^{2}\right)}\right) \exp \left(\frac{6 x^{2}}{(n+1)\left(1-m^{2}\right)}\right)$, whose derivative is:

$$
\begin{array}{r}
\left(\frac{1}{(n+1)\left(1-m^{2}\right)} P^{\prime}\left(\frac{x}{(n+1)\left(1-m^{2}\right)}\right)\right. \\
\left.+\frac{12 x}{(n+1)\left(1-m^{2}\right)} P\left(\frac{x}{(n+1)\left(1-m^{2}\right)}\right)\right) \exp \left(\frac{6 x^{2}}{(n+1)\left(1-m^{2}\right)}\right)
\end{array}
$$

At rank $k+1$ we have:

$$
\begin{array}{r}
b_{m, k+1}^{n}[p]=b_{m, k}^{n}[p]-b_{m, k}^{n}[p-1]=\sqrt{\frac{6}{\pi(n+1)\left(m^{2}-1\right)}} \times \\
\quad\left(\left[\exp \left(\frac{6 x^{2}}{(n+1)\left(1-m^{2}\right)}\right)\right]^{(k)}\left(p-\frac{k}{2}\right)\right. \\
=\sqrt{\frac{\left.\left.\exp \left(\frac{6 x^{2}}{(n+1)\left(1-m^{2}\right)}\right)\right]^{(k)}\left(p-1-\frac{k}{2}\right)\right)}{\pi(n+1)\left(m^{2}-1\right)} \exp \left(\frac{6\left(p-\frac{k+1}{2}\right)^{2}}{(n+1)\left(1-m^{2}\right)}\right) \times}
\end{array}
$$




$$
\begin{array}{r}
\left(P\left(\frac{p-\frac{k}{2}}{(n+1)\left(1-m^{2}\right)}\right) \exp \left(\frac{6\left(p-\frac{k}{2}-\frac{1}{4}\right)}{(n+1)\left(1-m^{2}\right)}\right)\right. \\
\left.-P\left(\frac{p-1-\frac{k}{2}}{(n+1)\left(1-m^{2}\right)}\right) \exp \left(\frac{-6\left(p-\frac{k}{2}-\frac{3}{4}\right)}{(n+1)\left(1-m^{2}\right)}\right)\right) \\
\approx \sqrt{\frac{6}{\pi(n+1)\left(m^{2}-1\right)}} \exp \left(\frac{6\left(p-\frac{k+1}{2}\right)^{2}}{(n+1)\left(1-m^{2}\right)}\right) \times \\
\left(P\left(\frac{p-\frac{k}{2}}{(n+1)\left(1-m^{2}\right)}\right)-P\left(\frac{p-1-\frac{k}{2}}{(n+1)\left(1-m^{2}\right)}\right)+\right. \\
\left.\frac{6\left(p-\frac{k}{2}-\frac{1}{2}\right)}{(n+1)\left(1-m^{2}\right)}\left(P\left(\frac{p-\frac{k}{2}}{(n+1)\left(1-m^{2}\right)}\right)+P\left(\frac{p-1-\frac{k}{2}}{(n+1)\left(1-m^{2}\right)}\right)\right)\right),
\end{array}
$$

using a first order approximation of the exponential,

$$
\begin{aligned}
& \approx \sqrt{\frac{6}{\pi(n+1)\left(m^{2}-1\right)}} \exp \left(\frac{6\left(p-\frac{k+1}{2}\right)^{2}}{(n+1)\left(1-m^{2}\right)}\right) \times \\
& \left(\frac{1}{(n+1)\left(1-m^{2}\right)} P^{\prime}\left(\frac{p-\frac{1+k}{2}}{(n+1)\left(1-m^{2}\right)}\right)\right. \\
& \left.+\frac{12 p-6(k+1)}{(n+1)\left(1-m^{2}\right)} P\left(\frac{p-\frac{1+k}{2}}{(n+1)\left(1-m^{2}\right)}\right)\right) \approx \\
& \sqrt{\frac{6}{\pi(n+1)\left(m^{2}-1\right)}} \\
& \times\left[\exp \left(-\frac{6 x^{2}}{(n+1)\left(m^{2}-1\right)}\right)\right]^{(k+1)}\left(p-\frac{k+1}{2}\right) .
\end{aligned}
$$

We now deal with the case $m$ even, for which we have:

$$
\begin{array}{r}
b_{m, k+1}^{n}[p]=b_{m, k}^{n}[p]-b_{m, k}^{n}[p-1]=\sqrt{\frac{6}{\pi(n+1)\left(m^{2}-1\right)}} \times \\
\left(\left[\exp \left(\frac{6 x^{2}}{(n+1)\left(1-m^{2}\right)}\right)\right]^{(k)}\left(p-\frac{k+(n+1)}{2}\right)\right. \\
\left.-\left[\exp \left(\frac{6 x^{2}}{(n+1)\left(1-m^{2}\right)}\right)\right]^{(k)}\left(p-1-\frac{k+(n+1)}{2}\right)\right) \\
=\sqrt{\frac{6}{\pi(n+1)\left(m^{2}-1\right)} \frac{12 \alpha}{(n+1)\left(1-m^{2}\right)} \times} \times \\
\text { for at least one } \alpha \in] p-1-\frac{k+n+1}{2}, p-\frac{k+n+1}{2} \\
{\left[\exp \left(\frac{6 x^{2}}{(n+1)\left(1-m^{2}\right)}\right)\right.}
\end{array}
$$


This completes the proof of Theorem 1 .

\section{APPENDIX B}

Proof of Proposition (6). We start with the case $n+k$ even. Since,

$$
\begin{aligned}
\alpha_{2, k}^{n}[p] & =b_{2, k}^{n}\left[p+\left\lfloor\frac{n+1+k}{2}\right\rfloor\right]=b_{2, k}^{n}\left[p+\frac{n+k}{2}\right] \\
& =\frac{1}{2}\left(b_{2, k}^{n-1}\left[p+\frac{n+k}{2}\right]+b_{2, k}^{n-1}\left[p-1+\frac{n+k}{2}\right]\right) \\
& =\frac{1}{2}\left(\alpha_{2, k}^{n-1}[p]+\alpha_{2, k}^{n-1}[p-1]\right)
\end{aligned}
$$

if we apply (5), we get:

$$
c_{2, k}^{n-1}=\frac{1}{2}\left(c_{2, k}^{n-1}[p]+c_{2, k}^{n-1}[p+1]\right) .
$$

The demonstration for the case $n+k$ odd is identical. We now prove the second equality of (6) when $n+k$ is even. Since we have:

$$
\begin{aligned}
\alpha_{2, k}^{n}[p] & =b_{2, k}^{n}\left[p+\left\lfloor\frac{n+1+k}{2}\right\rfloor\right]=b_{2, k}^{n}\left[p+\frac{n+k}{2}\right] \\
& =b_{2, k-1}^{n}\left[p+\frac{n+k}{2}\right]-b_{2, k-1}^{n}\left[p-1 \frac{n+k}{2}\right] \\
& =\alpha_{2, k-1}^{n}[p+1]-\alpha_{2, k-1}^{n}[p]
\end{aligned}
$$

by applying (6) we get:

$$
c_{2, k}^{n}[p]=c_{2, k-1}^{n}[p-1]-c_{2, k-1}^{n}[p]
$$

The demonstration when $n+k$ is odd is identical.

\section{REFERENCES}

[1] P. Abry and P. Flandrin, Multiresolution Transient Detection, In Proc. IEEE-SP Int, Symp. Time-Freq. Time-Scale Anal., Philadelphia, PA, pp. 225-228, 1994.

[2] K. Berkner and R. O. Wells, A New Hierarchical Scheme for Approximating the Continuous wavelet transform with Applications to Edge Detection, IEEE Signal Processing letters, vol. 6, no.8, pp. 1993-1995, 1999.

[3] K. Berkner and R.O. Wells, A Fast Approximation to the Continuous Wavelet Transform with Applications, Proceedings of the Asimolar, 1997.

[4] I. Daubechies, Ten Lectures on Wavelets, SIAM, 1992.

[5] M. Frish, H. Messer, The Use of the Wavelet Transform in the Detection of Unknown Transient Signal, IEEE Transactions on Information Theory, vol. 38, no. 2, pp. 893-897.

[6] M. Khalil, J. Duchêne, Detection and Classification of Multiple Events in Piecewise Stationary Signals: Comparison Between Autoregressive and Multiscale Approaches, Signal Processing, vol. 75, pp. 239-251, 1999.

[7] S.Mallat and S. Zhong, Characterization of Signals from Multiscale Edges, IEEE Trans. Pattern Anal. Mach. Intell., vol.14, pp. 710-732, 1992.

[8] S. Meignen, P-Y. Guméry, Contribution to the Berkner Scheme for the Approximation of the Continuous Wavelet Transform: Application to the Measure of the Latency Time of the Genioglossus Muscle, Revue Traitement du Signal, vol. 20, no. 4, pp. 375-388, 2003.

[9] L. Senhadji, J.J. Bellanger, G. Garrault, Time-scale Based Detection of Intercritical Paroxytic Events in Electroencephalography, Revue Traitement du Signal, vol. 12, pp. 357-371, 1995. 
[10] Z. Cvetkovic and M. Vetterli, OverSampled Filter Banks, IEEE Transactions on Signal Processing, vol. 46, no. 5, 1998.

[11] Yu-Ping Wang and S.L. Lee, Scale-space Derived from B-splines, IEEE Transactions on Pattern Analysis and Machine Intelligence, vol. 20, no. 10, pp. 1040-1055, 1998.

[12] A. Yuille and T. Poggio, Scaling Theorems for Zero-crossing, IEEE Transactions on Pattern Analysis and Machine Intelligence, vol. 8, no. 1, pp. 15-25, January 1986. 